\title{
A truss element for modelling reversible softening in living tissues
}

\author{
J.J. Muñoz ${ }^{1 a}$, V. Conte $^{b}$, N. Asadipour ${ }^{a}$, M. Miodownik ${ }^{b}$ \\ ${ }^{a}$ LaCàN, Dep. Appl. Math. III, Univ. Polit. de Catalunya (UPC), Barcelona, \\ Spain \\ ${ }^{b}$ Institute for Bioengineering of Catalonia (IBEC), Spain \\ ${ }^{c}$ Mechanical Engineering Dept, University College London, United Kingdom
}

\begin{abstract}
We resort to non-linear viscoelasticity to develop a truss element able to model reversible softening in lung epithelial tissues undergoing transient stretch. Such a Maxwell truss element is built by resorting to a three-noded element whose mid-node is kinematically constrained to remain on the line connecting the end-nodes. The whole mechanical system undergoes an additive decomposition of the strains along the truss direction where the total contribution of the mid-node is accounted for by using a null-space projection and static condensation techniques. Assembling of such line-elements in 3D networks allows us to model extended regions of living tissues as well as their anisotropies.
\end{abstract}

Keywords: Viscoelasticity, Maxwell, truss, null-space, reversible softening

\section{Introduction}

Latest developments in non-linear cell rheology reveal that cells respond with marked inelastic behaviour to externally-induced deformations [Trepat et al.(2007), Fernández and Ott(2008), Krishnan et al.(2009)], and that the cell activity may be responsible for such non-linearities [Banerjee and Marchetti(2012), MacKintosh and Levine(2008)]. Application of transient stretch to lung epithelial tissues, for instance, is reported to trigger cell softening followed by recovery of the initial cell stiffness [Trepat et al.(2007)], a process which will be here referred to as reversible softening. Mechanisms of inelastic cell deformation involving stiffening and softening are reported in the literature concerning several types of both cells [Fung(1993), Gavara et al.(2008), Krishnan et al.(2009)] and biopolymer networks [Janmey et al.(1991), Chaudhuri et al.(2007), Lin et al.(2011)Lin, Koenderink, and Weitz].

From the modelling point of view, material behaviour of this kind is clearly incompatible with classic linear viscoelasticity. Moreover, the highly

\footnotetext{
${ }^{1}$ j.munoz@upc.edu
} 
non-linear response of such tissues as well as the large deformations they undergo, represent a numerical challenge.

In this manuscript we analyse to what extent laws of non-linear elasticity and viscosity can be modified and combined in order to qualitatively reproduce softening and subsequent hardening of a truss element upon transient stretch. We could in principle do so by formulating the linear differential equation which opportunely describes the material system in the case of finite strains (e.g. [Holzapfel(2000), Ogden(1984), Simo(1987)]). The fibrous nature of some tissues can be handled by including the directions of the fibres in the strain-energy function (e.g. [Merodio and Ogden(2005), Merodio and Goicolea(2007), Merodio(2006)]) or directly in the stress field [Merodio and Rajagopal(2007)].

However, we perform the analysis by building a non-linear Maxwell element for living tissues because: (i) its viscoelastic rheological behaviour can be fully assigned by prescribing in an arbitrary way the elastic and viscous laws of its components; (ii) such components can be easily embedded in three-dimensional truss systems. Choice of specific elastic and viscous force vectors for this Maxwell truss element allows us to model the main features of fluidisation in lung epithelial tissues, as detailed in [Trepat et al.(2007)].

In order to better highlight the innovation of our Maxwell truss element for living tissues, in this paper we first present the implementation of a classic Maxwell model in linear viscoelasticity. Construction of the non-linear element is achieved by splitting the rheological law and by using null-space projection techniques - once non-linear constitutive laws for reversible fluidisation have been established. It is worth noticing that in our formulation we purposely disregard any process of cell active remodelling, commonly regarded as the ability of a cell to perform mechanical work at the expenses of internal sources of biochemical energy.

\section{Classic Maxwell rheological model}

The main assumption of the linear Maxwell model is that the viscous and elastic stresses are equal, i.e. $\sigma^{e}=\sigma^{v}=\sigma$ and are given by

$$
\sigma^{e}=k \varepsilon^{e} \quad, \quad \sigma^{v}=\eta \dot{\varepsilon}^{v}
$$

with $k$ and $\eta$ the material stiffness and viscosity, respectively. On the other hand, the strains must satisfy the following kinematic constraint:

$$
\dot{\varepsilon}=\dot{\varepsilon}^{e}+\dot{\varepsilon}^{v}
$$


with $\varepsilon$ the total strain. By combining Equations (1) and (2), we obtain the following linear evolution law,

$$
\dot{\sigma}+\frac{k}{\eta} \sigma=k \dot{\varepsilon}
$$

After time-discretising this equation, the resulting expression for $\sigma$ may be inserted in the equilibrium equations, and the current displacements at time $t_{n+1}$, denoted by $u_{n+1}$, are obtained, as originally implemented in [Cyr and Teter(1973), Zienkiewicz(1978)].

The extension of the outlined procedure to multidimensional finite-strain viscoelasticity is usually achieved by replicating the linear evolution law in (3) with stress-like internal variables [Holzapfel(2000), Lubliner(2008), Simo(1987), Valanis(1972)], or for the analysis on a continuous spectrum and using quasi-linear viscoelasticity [Fung(1993)], numerically approximating the convolution integral [Puso and Weiss(1998)]. However, in some situations, the linear evolution law in (3) may not be sufficient to simulate the viscoelastic response of living tissues. For instance, it has been experimentally observed that after an imposed strain cycle, the cell viscosity decreases and it is eventually recovered [Trepat et al.(2007)]. These kind of non-linearities in the viscous contribution have motivated the methodology presented in this work.

\section{Rheological splitting and null-space projection}

Let us define the configuration of an arbitrary truss element through the positions of the two end points. At the initial (assumed stress-free) and current configurations, they are respectively given by the pairs $\left(\boldsymbol{X}_{1}, \boldsymbol{X}_{2}\right)$ and $\left(\boldsymbol{x}_{1}, \boldsymbol{x}_{2}\right)$. In order to construct a Maxwell element, we split the elastic and viscous components along the truss direction into two distinct elements, in the same manner as it is done in Eq. (2). Accordingly, the whole element joining nodes $\boldsymbol{x}_{1}$ and $\boldsymbol{x}_{2}$ is assigned an additional intermediate node $\boldsymbol{x}_{3}$ that determines the elastic component (between nodes $\boldsymbol{x}_{1}$ and $\boldsymbol{x}_{3}$ ), and the viscous component (between nodes $\boldsymbol{x}_{3}$ and $\boldsymbol{x}_{2}$ ), as shown in Figure 1a. The elastic and viscous components are then allowed to have any general constitutive law. We point out that the kinematic splitting of the strains described here is equivalent to the deformation gradient decomposition in finite strains for viscoelastic models (see [Gasser and Forsell(2011)]). In our case though, the elastic and viscous parts are handled independently, with no evolution law that couples them, and are mechanically and algebraically merged into a single element using the methodology described next. 


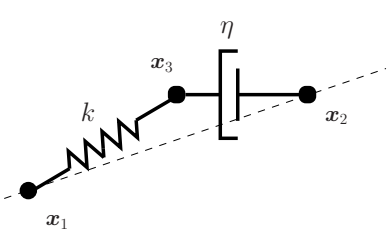

(a)

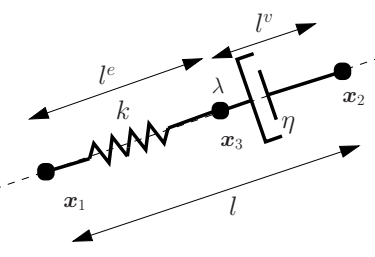

(b)

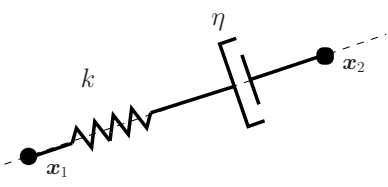

(c)

Figure 1: Representation of construction process of Maxwell element: (a) initial 2 elements, (b) constrained 2 elements where node 3 is enforced to move along line between nodes 1 and 2, and (c) final element after static condensation of internal dof $\lambda$.

As indicated in Figure 1b, node $\boldsymbol{x}_{3}$ is constrained to move along the line joining nodes 1 and 2 . Consequently, its position may be then described by using an internal variable time dependent $\lambda$ and the following equation:

$$
\boldsymbol{x}_{3}=\boldsymbol{x}_{1}+\lambda\left(\boldsymbol{x}_{2}-\boldsymbol{x}_{1}\right)
$$

Without loss of generality, $\boldsymbol{x}_{3}$ can be selected arbitrarily between $\boldsymbol{x}_{1}$ and $\boldsymbol{x}_{3}$, and that the present work sets its initial position in the middle between $\boldsymbol{x}_{1}$ and $\boldsymbol{x}_{2}$, i.e. $\lambda_{0}=0.5$. We have numerically verified that the value of $\lambda_{0}$ does not affect the resulting elastic and viscous strains and stresses, to be defined next. The total initial (stress-free) and total current lengths of the truss are obtained as $L=\left\|\boldsymbol{X}_{2}-\boldsymbol{X}_{1}\right\|$ and $l=\left\|\boldsymbol{x}_{2}-\boldsymbol{x}_{1}\right\|$, respectively, while the elastic and viscous lengths of the truss will be denoted by $l^{e}=\left\|\boldsymbol{x}_{1}-\boldsymbol{x}_{3}\right\|$ and $l^{v}=\left\|\boldsymbol{x}_{2}-\boldsymbol{x}_{3}\right\|$. The scalar elastic and viscous strains are defined by,

$$
\varepsilon^{e}=\frac{l^{e}-L^{e}}{L} \quad, \quad \varepsilon^{v}=\frac{l^{v}-L^{v}}{L} .
$$

which satisfy the additive decomposition $\varepsilon=\varepsilon^{e}+\varepsilon^{v}$, with $\varepsilon=(l-L) / L$. These strain measures are evaluated along the arbitrary truss direction, so that the relations between the length measures $l^{e}$ and $l^{v}$, and the nodal positions $\boldsymbol{x}_{i}$ are non-linear. Therefore, the strain measures correspond to non-linear co-rotated strains which can handle large truss displacements and rotations. Furthermore, since the value of $l$ remains unchanged after applying a rigid body motion, the strain measures employed are objective.

We next transform the constrained system of the two trusses into an unconstrained system by resorting to the so-called null-space (or masterslave) method, extensively employed in mechanical systems (see for instance [Muñoz and Jelenić(2004), Betsch(2005)]). In this manner, the system described by the nodal positions $\boldsymbol{x}_{1}, \boldsymbol{x}_{2}$ and $\boldsymbol{x}_{3}$ is analysed as a function of the 
independent variables $\boldsymbol{x}_{1}, \boldsymbol{x}_{2}$ and the parameter $\lambda$. Furthermore, since the parametric position $\lambda$ is an elemental internal variable, it can be statically condensed in the solution process [Bathe(1996)]. As a result, a two-noded element which can handle general elastic and viscous constitutive laws is constructed. The application of null-space to the present two-truss system is described in detail in the Appendix.

\section{Constitutive laws for reversible softening}

We propose viscoelastic constitutive laws that aim to model the observed mechanical response of the cell tissue, associated with both the active and passive interaction of all the structural elements in the cytoskeleton (actin fibers, myosin, cross-links, intermediate and apical filaments, microtubules,...). We determine such non-linear constitutive laws for our truss element by modifying their linear counterpart respectively. The equation governing the Maxwell truss element for living tissues is finally obtained by combining the viscous and elastic non-linear components as detailed above.

\subsection{Elastic element with linear law}

The total elastic energy of a truss of total initial length $L$ is determined in this case by the following quadratic potential energy,

$$
V\left(\varepsilon^{e}\right)=\frac{L}{2} k\left(\varepsilon^{e}\right)^{2},
$$

with $\varepsilon^{e}$ the elastic strain defined in Eq. (5). We note that since $L=$ $\left\|\boldsymbol{X}_{1}-\boldsymbol{X}_{2}\right\| \neq L^{e}=\left\|\boldsymbol{X}_{1}-\boldsymbol{X}_{3}\right\|$, the elastic strain $\varepsilon^{e}$ in (5) differs from the total strain, and also from the standard strain of the reduced element between node $\boldsymbol{x}_{1}$ and $\boldsymbol{x}_{3}$, which is given by $\varepsilon^{*}=\left(l^{e}-L^{e}\right) / L^{e}$. The relation between the latter and $\varepsilon^{e}$ may be expressed as

$$
\varepsilon_{n+1}^{e}=\frac{l_{n+1}^{e}-L^{e}}{L}=\lambda_{0} \frac{l_{n+1}^{e}-L^{e}}{L^{e}}=\lambda_{0} \varepsilon_{n+1}^{*} .
$$

For clarity, in the remaining expressions, we will remove the subscript $n+1$. The elastic stress is given by $\sigma^{e}=\partial V / \partial \varepsilon^{e}=k \varepsilon^{e}$, while the elastic contribution to the truss residual, computed as $\hat{\boldsymbol{g}}^{e}=\nabla_{x} V$, is explicitly given by,

$$
\hat{\boldsymbol{g}}^{e}=k \varepsilon^{e} \boldsymbol{e}_{13}=\left\{\begin{array}{l}
\hat{\boldsymbol{g}}_{1}^{e} \\
\hat{\boldsymbol{g}}_{3}^{e}
\end{array}\right\}, \text { with } \boldsymbol{e}_{i j}=\frac{1}{\left\|\boldsymbol{x}_{i}-\boldsymbol{x}_{j}\right\|}\left\{\begin{array}{l}
\boldsymbol{x}_{i}-\boldsymbol{x}_{j} \\
\boldsymbol{x}_{j}-\boldsymbol{x}_{i}
\end{array}\right\}
$$




\subsection{Elastic element with exponential law}

It has been observed in vitro that the tissue stiffness decreases as the applied load increases, but contrarily to what occurs to non-living materials, it is able to recover its initial value as the load is removed in a fully reversible manner [Chaudhuri et al.(2007)]. Also, after applying a stretch cycle to a tissue, the material stiffness and viscosity decrease, but are eventually recovered [Trepat et al.(2007)], a response that is characteristic of a fluidisation process. In order to account for these recoverable and reversible softening, we postulate an elastic potential of the type:

$$
V\left(\varepsilon^{e}\right)=\frac{L k_{0}}{2 \alpha}\left(1-e^{-\alpha\left(\varepsilon^{e}\right)^{2}}\right),
$$

with $\alpha \geq 0$ a material parameter. According to Eq. (8), the stored elastic energy is bounded by the value $\frac{L k_{0}}{2 \alpha}$. The corresponding elastic stress is given by,

$$
\sigma_{e l}=\frac{\partial V}{\partial \varepsilon^{e}}=k_{0} \varepsilon^{e} e^{-\alpha\left(\varepsilon^{e}\right)^{2}}
$$

so that the elastic stress does not monotonically increase, but is eventually reduced as $\varepsilon^{e}$ increases. At a given strain, the non-linear effective stiffness of the material can be computed as $k=\frac{\partial \sigma}{\partial \varepsilon}=k_{0} e^{-\alpha\left(\varepsilon^{e}\right)^{2}}\left(1-2 \alpha\left(\varepsilon^{e}\right)^{2}\right)$, which decreases as the elastic strain increases, and in a reversible manner as shown in [Chaudhuri et al.(2007)]. We do not attempt to physically explain at the nano-scale the source of this softening, but we do aim to test rheological laws that can reproduce at the micro-scale such behaviour.

The contribution to the residual of an elastic truss with nodal ends in $\boldsymbol{x}_{1}$ and $\boldsymbol{x}_{3}$ is given by $\nabla_{x} V$, that is,

$$
\hat{\boldsymbol{g}}^{e}=k_{0} \varepsilon^{e} e^{-\alpha\left(\varepsilon^{e}\right)^{2}} \boldsymbol{e}_{13}
$$

\subsection{Viscous element with linear law}

In linear $1 \mathrm{D}$ viscosity, the viscous stress is equal to $\sigma^{v}=\eta_{0} \dot{\varepsilon}^{v}$, with $\eta_{0}$ the material viscous coefficient and $\varepsilon_{n+1}^{v}=\frac{l_{n+1}^{v}-L^{v}}{L}$ the viscous strain. In a general truss element, the viscous nodal forces are approximated by,

$$
\hat{\boldsymbol{g}}^{v}=\eta_{0} \dot{\varepsilon}^{v} \boldsymbol{e}_{32} .
$$

This contribution will be discretised in time by employing the time-step size $\Delta t=t_{n+1}-t_{n}$ and the following time-stepping,

$$
\hat{\boldsymbol{g}}_{n+\theta}^{v}=\eta_{0} \frac{l_{n+1}^{v}-l_{n}^{v}}{L \Delta t} \boldsymbol{e}_{32, n+\theta}=\lambda_{0} \eta_{0} \frac{l_{n+1}^{v}-l_{n}^{v}}{L^{v} \Delta t} \boldsymbol{e}_{32, n+\theta},
$$


where

$$
\begin{aligned}
l_{n+\theta}^{v} & =\left\|\boldsymbol{x}_{2, n+\theta}-\boldsymbol{x}_{3, n+\theta}\right\|, & \boldsymbol{x}_{i, n+\theta}=(1-\theta) \boldsymbol{x}_{i, n}+\theta \boldsymbol{x}_{i, n+1} \\
\hat{\boldsymbol{g}}_{n+\theta}^{v} & =(1-\theta) \boldsymbol{g}_{n}^{v}+\theta \boldsymbol{g}_{n+1}^{v}, & \boldsymbol{e}_{32, n+\theta}=\frac{1}{l_{n+\theta}^{v}}\left\{\begin{array}{l}
\boldsymbol{x}_{3}-\boldsymbol{x}_{2} \\
\boldsymbol{x}_{2}-\boldsymbol{x}_{3}
\end{array}\right\}_{n+\theta},
\end{aligned}
$$

and with $\theta \in[0,1]$ an algorithmic parameter. From Eq. (12), it follows that the load contribution at time $t_{n+1}$, to be assembled together with the elastic part deduced in Sections 4.1 or 4.2 , reads

$$
\hat{\boldsymbol{g}}_{n+1}^{v}=\frac{\theta-1}{\theta} \boldsymbol{g}_{n}^{v}+\frac{\eta_{0}}{\theta L} \frac{l_{n+1}^{v}-l_{n}^{v}}{\Delta t} \boldsymbol{e}_{32, n+\theta}=\left\{\begin{array}{c}
\hat{\boldsymbol{g}}_{3}^{v} \\
\hat{\boldsymbol{g}}_{2}^{v}
\end{array}\right\}
$$

\subsection{Viscous element with exponential law}

Similarly to the elastic case, it has been experimentally observed that the material viscosity may decresase after an imposed stretching process [Trepat et al.(2007)], and that after a given period of time (independent of the amount of prestretch), the material recovers the initial elastic and viscous properties. In order to mimic such a behaviour at the micro-scale level, we suggest the following non-linear viscous law:

$$
\hat{\boldsymbol{g}}^{v}=\eta_{0} e^{-\beta\left(\varepsilon^{v}\right)^{2}} \dot{\varepsilon}^{v} \boldsymbol{e}_{32}
$$

In this case, the factor $\eta=\eta_{0} e^{-\beta\left(\varepsilon^{v}\right)^{2}}$ has the role of a strain-dependent effective viscosity that decreases as the viscous strain increases. The time integration of the previous equation is resolved resorting again to a $\theta$-averaged time-stepping,

$$
\hat{\boldsymbol{g}}_{n+\theta}^{v}=\eta_{0} e^{-\beta\left(\varepsilon_{n+\theta}^{v}\right)^{2}} \frac{\Delta \varepsilon^{v}}{\Delta t} \boldsymbol{e}_{32, n+\theta}
$$

\subsection{Maxwell truss element for living tissues}

The element employed in our numerical results is the result of assembling the elastic and viscous residual contributions in Eqns. (10) and (14), and projected with the null-space method,

$$
\mathbf{N}^{T}\left\{\begin{array}{l}
\boldsymbol{g}_{1}^{e} \\
\boldsymbol{g}_{3}^{e}+\boldsymbol{g}_{3}^{v} \\
\boldsymbol{g}_{2}^{v}
\end{array}\right\}
$$


The vectors $\boldsymbol{g}_{i}^{e}$ and $\boldsymbol{g}_{i}^{v}$ are the nodal contributions of the elastic and viscous forces. The explicit expression of matrix $\mathbf{N}$, which imposes the nullspace projection, is deduced in Eq. (20), which after performing the matrix multiplication in (15) give rise to the following residual:

$$
\tilde{\boldsymbol{g}}=\left\{\begin{array}{l}
\tilde{\boldsymbol{g}}_{1} \\
\tilde{g}_{\lambda} \\
\tilde{\boldsymbol{g}}_{2}
\end{array}\right\}=\left\{\begin{array}{l}
\boldsymbol{g}_{1}^{e}+(1-\lambda)\left(\boldsymbol{g}_{3}^{e}+\boldsymbol{g}_{3}^{v}\right) \\
\left(\boldsymbol{x}_{2}-\boldsymbol{x}_{1}\right)^{T}\left(\boldsymbol{g}_{3}^{e}+\boldsymbol{g}_{3}^{v}\right) \\
\boldsymbol{g}_{2}^{v}+\lambda\left(\boldsymbol{g}_{3}^{e}+\boldsymbol{g}_{3}^{v}\right)
\end{array}\right\}
$$

This force vector depends on the internal dof $\lambda$, which is statically condensed, so that a two-noded element with arbitrary elastic and viscous laws is obtained. The expression of this residual two-noded residual depends on the Jacobian matrix of the force vector in (16), which we have not explicitly written here, but is computed using standard derivations. For more details on the static condensation see for instance [Bathe(1996)]. We also point out that in our case, by setting $\alpha=0$ or $\beta=0$ we recover the linear elastic law in Eq. (7) or the linear viscous law in Eq. (11).

\section{$5 \quad$ Numerical Results}

In the subsequent examples the non-linear equations are solved using a fully Newton-Raphson method with $\theta=0.5$, which has ensured numerically stable results. In all cases, we have used the reference value $k_{0}=1$, and in the one truss example in Section 5.1, we have used the value $\eta_{0}=$ 4 , which agrees with the relation $\eta_{0} / k_{0}=4$ measured experimentally in [Trepat et al.(2007)]. We have fixed the left boundary and prescribed the displacements at the right boundary according to the function $\bar{u}$ shown in Figure 2a.

\subsection{Stress relaxation of single non-linear Maxwell element}

We have first analysed the Maxwell element in Figure 2a with the values $\alpha=1,10,20,30$ and $\beta=0$. As indicated in Eq. (9), the effective stiffness $k=k_{0} e^{-\alpha\left(\varepsilon^{e}\right)^{2}}\left(1-2 \alpha\left(\varepsilon^{e}\right)^{2}\right)$ decreases as $\varepsilon^{e}$ increases, and this reduction is more pronounced as the material parameter $\alpha$ increases. For $t>2$ the global truss remains undeformed while the stress recovers asymptotically to its original value after approximately one time unit.

In order to also model the reversible drop in the viscosity, we applied the non-linear viscous law given in (11) with $\beta=0$ (linear viscosity) and $\beta=100$, while using $\alpha=30$ in both cases. Figure 3 shows the evolution of 


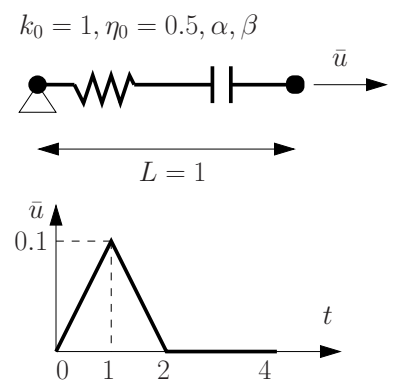

(a)

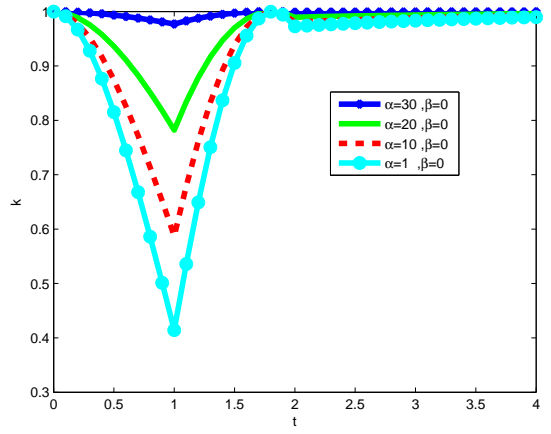

(b)

Figure 2: 1 truss element with constrained displacements. (a) Geometry, material properties and boundary conditions. (b) Value of the effective stiffness $k=k_{0} e^{-\alpha\left(\varepsilon^{e}\right)^{2}}\left(1-2 \alpha\left(\varepsilon^{e}\right)^{2}\right)$.

the effective stiffness and viscosity. When $\beta=100$, the viscosity is reduced by more than $50 \%$ with respect to the analysis with $\beta=0$. More importantly, the variation of $\beta$ has minimal effects on the evolution of the effective stiffness, which allows us to fit the parameters $\alpha$ and $\beta$ independently.

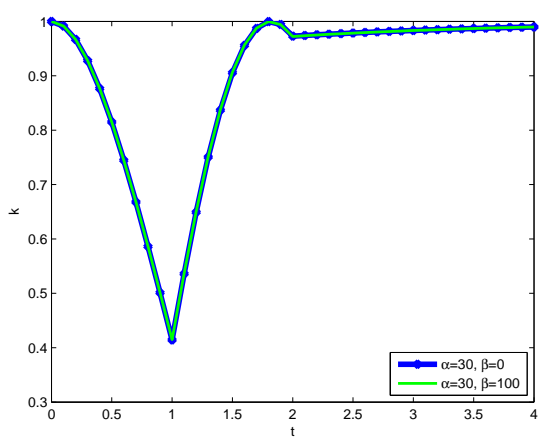

(a)

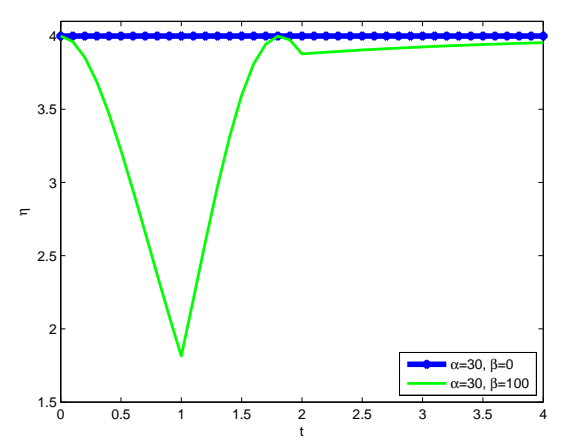

(b)

Figure 3: 1 truss element with non-linear viscosity. Evolution of (a) the effective stiffness $k=k_{0} e^{-\alpha\left(\varepsilon^{e}\right)^{2}}\left(1-2 \alpha\left(\varepsilon^{e}\right)^{2}\right)$ and (b) the effective viscosity $\eta=\eta_{0} e^{-\beta\left(\varepsilon^{v}\right)^{2}}$.

We have also attempted to reproduce the evolution of the phase angle $\delta=\tan ^{-1}(k / \eta)$ for the Maxwell element, and for different values of the maximum displacement. Figure 4a shows the measured evolution, while Figure $4 \mathrm{~b}$ shows the evolution after applying the strain during the first 2 
units of time (no experimental data is available for the evolution of $\delta$ while the material is stretched). We have used the parameters $\alpha=10$ and $\beta=400$, with the same values of $k$ and $\eta_{0}$ previously employed. It can be observed that the numerical and experimental curves exhibit similar trends, although in our numerical experiments the recovery time is much shorter. Current work is being performed in order to obtain a more accurate recovery time.

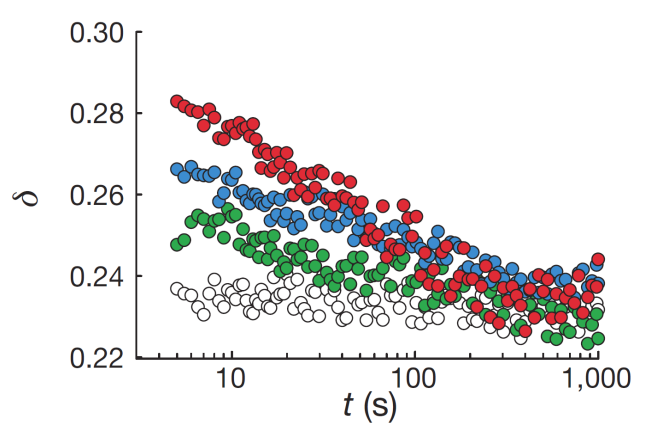

(a)

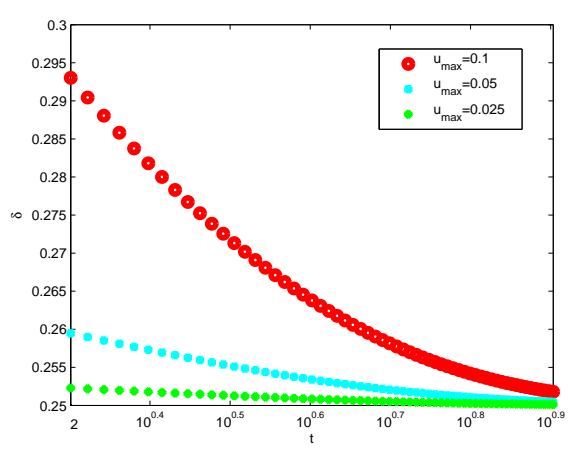

(b)

Figure 4: Experimental evolution of the phase angle after applying the stretch cycle, and numerical results when using one element for different values of maximum displacement.

\subsection{D tissues}

Embedding of the Maxwell truss element in 3D networks allows simulation of non-linear response of 3D domains of tissue under large strains and rotations in the real space. Cells are highly inhomogeneous and intrinsically discontinuous from the material point of view, as their interior architecture shows a very high degree of differentiation. Cellular tissues modelled as networks of trusses may be therefore thought of as a simplified version of generalised continua [Altenbach et al.(2011)], where bending stiffness is disregarded and no rotational degree of freedom (dof) is accounted for. However, use of a truss system shows essential differences compared to the use of a continuum. In isotropic linear elasticity, for instance, continua are characterised by two material parameters whereas only one is necessary to fully assign constitutive behaviour of any truss system. Moreover, the Poisson effect in truss systems strongly depends on the topology of the network (e.g. [Hahn et al.(2010)Hahn, Wallmersperger, and Kröplin]). Thus Truss systems are particularly appealing for analysing polymer-like networks (e.g. 
[Kim et al.(2009), Cyron and Wall(2010)]), especially if one takes into account the ease and efficiency with which general non-linear laws of both elasticity and viscosity may be combined in the Maxwell truss element detailed in this work.

With these ideas in mind we built a 3D system of non-linear Maxwell trusses that aims to emulate the global cytoskeletal actino-myosin network spreading across epithelial tissues through cell-cell junctions. Elastic softening with elemental parameter $\alpha=100$ has been adopted for each single truss element in the network (see Section 5.1). The epithelial cell patch is assumed to have generic dimensions $1.0 \times 1.0 \times 0.0625$ and formed by $E=2473$ trusses (Fig.5). We have run two analyses, one with prescribed displacements $\bar{u}_{x}=\bar{u}$ and another with $\bar{u}_{x}=\bar{u} / 2$.

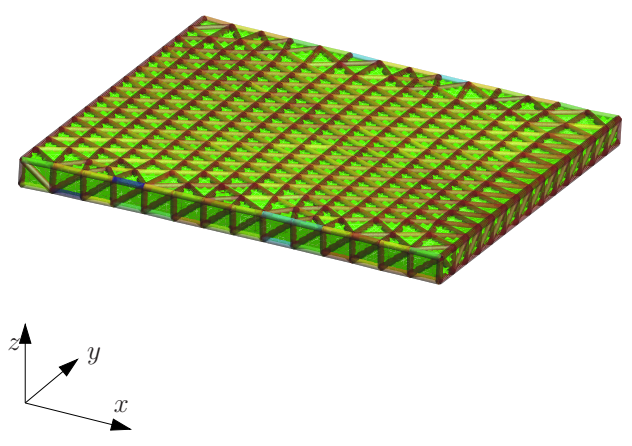

(a)

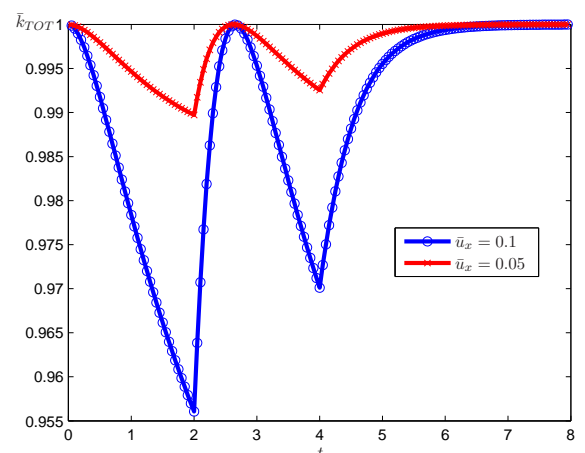

(b)

Figure 5: (a) Deformed configuration at $t=1$, with values of $k=k_{0} e^{-\alpha\left(\varepsilon^{e}\right)^{2}}$ (b), where dark red indicates $k=1$ and dark blue indicates $k \approx 0.5$. (b) Evolution of total effective stiffness $k_{T O T}$.

Due to the stretching, the epithelium undergoes a softening effect in the $x$ direction. Fig. 5a shows the contour plot of the values of the effective stiffness $k=k_{0} e^{-\alpha\left(\varepsilon^{e}\right)^{2}}\left(1-2 \alpha\left(\varepsilon^{e}\right)^{2}\right)$. As expected, those trusses aligned to the $x$ direction have their effective stiffness further reduced than those aligned to the $y$ or $z$ direction.

Figure $5 \mathrm{~b}$ shows for the two values of the prescribed displacements, the evolution of the averaged total effective stiffness $k_{T O T}$, computed as $\bar{k}_{T O T}=\left(\sum_{i=1}^{E} k^{i}\right) / E$, with $k^{i}$ the effective stiffness of truss $i$. Although the boundary conditions are similar to those in the previous example, the reduction in $\bar{k}_{T O T}$ is far smaller in the present case. This is due to the fact that the effective stiffness is averaged among all trusses, regardless of their orientation, while the applied strain is highly anisotropic. In fact, dur- 
ing the stretching process, some trusses have their effective stiffness reduced $49 \%$, while the stiffness of other trusses, mostly perpendicular to the stretch, remains nearly unaffected.

\section{Conclusions}

We have presented a general methodology for modelling non-linear viscoelastic materials through systems of Maxwell truss elements. The proposed technique makes it easy to handle any non-linear viscoelastic constitutive law. The model has been developed to simulate the non-linear mechanical response of actin-networks in soft tissues but can be equally applied to other engineering problems, and may be easily extended to include other non-linear effects such as incompressibility constraints or growth. When applied to reversible fluidisation in lung epithelial tissues subjected to transient stretch [Trepat et al.(2007)], the model shows that elastic and viscous non-linear laws can be suitably combined in order to qualitatively reproduce the behaviour of living materials without accounting for any cellular active remodelling.

\section{Acknowledgements}

Vito Conte has been financially supported by EMBO, under the Short Term Fellowship ASTF 203.00-2010, entitled "Modelling the viscoelastic behaviour of human lung epithelial cells." The authors are also very thankful to X Trepat at IBEC, Spain, for his fruitful discussions and scientific support.

\section{A Null-space projection}

We will assume that for each truss, the elastic and viscous nodal forces are contained in the vectors $\hat{\boldsymbol{g}}^{e}$ and $\hat{\boldsymbol{g}}^{v}$, which after the assembling process yield the general nodal contributions $\hat{\boldsymbol{g}}$. These vectors may be written as,

$$
\hat{\boldsymbol{g}}^{e}=\left\{\begin{array}{l}
\hat{\boldsymbol{g}}_{1}^{e} \\
\hat{\boldsymbol{g}}_{3}^{e}
\end{array}\right\}, \hat{\boldsymbol{g}}^{v}=\left\{\begin{array}{l}
\hat{\boldsymbol{g}}_{3}^{v} \\
\hat{\boldsymbol{g}}_{2}^{v}
\end{array}\right\}, \hat{\boldsymbol{g}}=\left\{\begin{array}{l}
\boldsymbol{g}_{1}^{e} \\
\boldsymbol{g}_{3}^{e}+\boldsymbol{g}_{3}^{v} \\
\boldsymbol{g}_{2}^{v}
\end{array}\right\}=\left\{\begin{array}{l}
\hat{\boldsymbol{g}}_{1} \\
\hat{\boldsymbol{g}}_{3} \\
\hat{\boldsymbol{g}}_{2}
\end{array}\right\},
$$

The vector $\hat{\boldsymbol{g}}$ is conjugate to the virtual displacement vector $\delta \boldsymbol{x}_{132}^{T}=$ $\left\{\delta \boldsymbol{x}_{1}^{T}, \delta \boldsymbol{x}_{3}^{T}, \delta \boldsymbol{x}_{2}^{T}\right\}$, and the total work (elastic and dissipated in the viscous 
element) is then expressed as:

$$
\mathcal{W}=\delta \boldsymbol{x}_{132} \cdot \hat{\boldsymbol{g}}
$$

On the other hand, the kinematic constraint in Eq. (4) induces the following relation between the virtual counterparts:

$$
\delta \boldsymbol{x}_{3}=(1-\lambda) \delta \boldsymbol{x}_{1}+\left(\boldsymbol{x}_{2}-\boldsymbol{x}_{1}\right) \delta \lambda+\lambda \delta \boldsymbol{x}_{2} .
$$

which allows us to write the following relation between the unconstratined $\operatorname{dof} \delta \boldsymbol{x}_{1 \lambda 2}^{T}=\left\{\delta \boldsymbol{x}_{1}^{T} \delta \lambda \delta \boldsymbol{x}_{2}^{T}\right\}$ and $\delta \boldsymbol{x}_{132}$ :

$$
\delta\left\{\begin{array}{l}
\boldsymbol{x}_{1} \\
\boldsymbol{x}_{3} \\
\boldsymbol{x}_{2}
\end{array}\right\}=\left[\begin{array}{ccc}
\mathbf{I} & \mathbf{0} & \mathbf{0} \\
(1-\lambda) \mathbf{I} & \boldsymbol{x}_{2}-\boldsymbol{x}_{1} & \lambda \mathbf{I} \\
\mathbf{0} & \mathbf{0} & \mathbf{I}
\end{array}\right] \delta\left\{\begin{array}{c}
\boldsymbol{x}_{1} \\
\lambda \\
\boldsymbol{x}_{2}
\end{array}\right\}=\mathbf{N} \delta\left\{\begin{array}{c}
\boldsymbol{x}_{1} \\
\lambda \\
\boldsymbol{x}_{2}
\end{array}\right\} .
$$

The total virtual work in (18) may be now re-expressed as,

$$
\delta \mathcal{W}=\delta \boldsymbol{x}_{1 \lambda 2} \cdot \mathbf{N}^{T} \hat{\boldsymbol{g}}=\delta \boldsymbol{x}_{1 \lambda 2} \cdot \tilde{\boldsymbol{g}}
$$

where matrix $\mathbf{N}$ projects the constrained residual $\hat{\boldsymbol{g}}$ onto the new residual $\tilde{\boldsymbol{g}}:=\mathbf{N}^{T} \hat{\boldsymbol{g}}$, which is conjugate to the unconstrained variables $\delta \boldsymbol{x}_{1 \lambda 2}$.

\section{References}

[Altenbach et al.(2011)] Altenbach, H., Maugin, G., Erofeev, V., 2011. Mechanics of generalized continua, 1st Edition. Advanced Structured Materials. Springer-Verlag, Berlin.

[Banerjee and Marchetti(2012)] Banerjee, S., Marchetti, M., 2012. Contractile stresses in cohesive cell layers on finite-thickness substrates. Phys. Rev. LettersIn press. http://arxiv.org/pdf/1204.3019v3.pdf.

[Bathe(1996)] Bathe, K., 1996. Finite Element Procedures. Prentice Hall.

[Betsch(2005)] Betsch, P., 2005. The discrete null space method for the energy consistent integration of constrained mechanical systems. Part I: Holonomic constraints. Comp. Meth. Appl. Mech. Engng. 194, 51595190 .

[Chaudhuri et al.(2007)] Chaudhuri, O., Parekh, S., Fletcher, D., 2007. Reversible stress softening of actin networks. Nature 445, 295-298. 
[Cyr and Teter(1973)] Cyr, N. A., Teter, R. D., 1973. Finite element elastic plastic creep analysis of two dimensional continuum with temperature dependent material properties. Comput. Struct. 3, 849-863.

[Cyron and Wall(2010)] Cyron, C. J., Wall, W. A., 2010. Consistent finiteelement approach to Brownian polymer dynamics with anisotropic friction. Phys. Rev. E 82 (066705).

[Fernández and Ott(2008)] Fernández, P., Ott, A., 2008. Single cell mechanics: stress stiffening and kinematic hardening. Phys. Rev. Letters 100, 238102 .

[Fung(1993)] Fung, Y. C., 1993. Biomechanics : mechanical properties of living tissues, 2nd Edition. Springer, New York.

[Gasser and Forsell(2011)] Gasser, T., Forsell, C., 2011. The numerical implementation of invariant-based viscoelastic formulations at finite strains. An anisotropic model for the passive myocardium. Comp. Meth. Appl. Mech. Engng. 200, 3637-3645.

[Gavara et al.(2008)] Gavara, N., Roca-Cusachs, P., Sunyer, R., Farre, R., Navajas, D., 2008. Mapping cell-matrix stresses during stretch reveals inelastic reorganization of the cytoskeleton. Bioph. J. 95, 464-471.

[Hahn et al.(2010)Hahn, Wallmersperger, and Kröplin] Hahn, _ M., Wallmersperger, T., Kröplin, B. H., 2010. Discrete element representation of continua: Proof of concept and determination of the material parameters. Comp. Mat. Sc. 50, 391-402.

[Holzapfel(2000)] Holzapfel, G. A., 2000. Nonlinear solid mechanics. A continuum approach for engineers. J Wiley \& Sons Ltd.

[Janmey et al.(1991)] Janmey, P. A., Euteneuer, U., Traub, P., Schliwa, M., 1991. Viscoelastic properties of vimentin compared with other filamentous biopolymer networks. J. Cell Biol. 113 (20), 155-160.

[Kim et al.(2009)] Kim, T., Hwang, W., Lee, H., Kamm, R. D., 2009. Computational analysis of viscoelastic properties of crosslinked actin networks. PLOS Comp. Biol. 5 (7), 1-13.

[Krishnan et al.(2009)] Krishnan, R., Park, C. Y., Lin, Y. C., Mead, J., Jaspers, R. T., Trepat, X., Lenormand, G., Tambe, D., Smolensky, A. V., Knoll, A. H., Butler, J. P., Fredberg, J. J., 2009. Reinforcement 
versus fluidization in cytoskeletal mechanoresponsiveness. PLOS ONE 4 , e5486.

[Lin et al.(2011)Lin, Koenderink, and Weitz] Lin, Y. C., Koenderink, G. H., Weitz, F. C. M. D. A., 2011. Control of non-linear elasticity in F-actin networks with microtubules. Soft Matter 7, 902-906.

[Lubliner(2008)] Lubliner, J., 2008. Plasticity theory, 3rd Edition. Dover Publ., dover edit. Mcmillan ed. in 1990.

[MacKintosh and Levine(2008)] MacKintosh, F., Levine, A. J., 2008. Nonequilibrium mechanics and dynamics of motor-activated gels. Phys. Rev. Letters 100, 018104.

[Merodio(2006)] Merodio, J., 2006. On constitutive equations for fiberreinforced nonlinearly viscoelastic solids. Mech. Res. Comm. 33, 764770 .

[Merodio and Goicolea(2007)] Merodio, J., Goicolea, J., 2007. On thermodynamically consistent constitutive equations for fiber-reinforced nonlinearly viscoelastic solids with application to biomechanics. Mech. Res. Comm. 34, 561-571.

[Merodio and Ogden(2005)] Merodio, J., Ogden, R., 2005. Tensile instabilities and ellipticity in fiber-reinforced compressible non-linearly elastic solids. Int. J. Eng. Sc. 43, 697-706.

[Merodio and Rajagopal(2007)] Merodio, J., Rajagopal, K., 2007. Constitutive equations for anisotropic nonlinearly viscoelastic materials. Mathem. Mech. Solids 34, 131-147.

[Muñoz and Jelenić(2004)] Muñoz, J. J., Jelenić, G., 2004. Sliding contact conditions using the master-slave approach with application on the geometracally non-linear beams. Int. J. Solids Struct. 41, 6963-6992.

[Ogden(1984)] Ogden, R., 1984. Non-linear elastic deformations. Vol. 26. Dover Publications.

[Puso and Weiss(1998)] Puso, M. A., Weiss, J. A., 1998. Finite element implementation of anisotropic quasi-linear viscoelasticity using a discrete spectrum approximation. J. Biomech. Engin. 120, 62-70.

[Simo(1987)] Simo, J. C., 1987. On a fully three-dimensional finite-strain viscoelastic damage model: Formulation and computational aspects. Comp. Meth. Appl. Mech. Engng. 60, 153-173. 
[Trepat et al.(2007)] Trepat, X., Deng, L., An, S., Navajas, D., Tschumperlin, D., Gerthoffer, W., Butler, J., Fredberg, J., 2007. Universal physical responses to stretch in the living cell. Nature 447 (3), 592-596.

[Valanis(1972)] Valanis, K., 1972. Irreversible thermodynamics of continuous media, Internal variable theory. Vol. 77 of CISM Courses and Lectures. Springer-Verlag, Internatinal center for mechanical sciences, Wien.

[Zienkiewicz(1978)] Zienkiewicz, O. C., 1978. Computational Mechanics, Ed: J T Oden. TICOM Lecture Notes on Matehmatics, 461. SpringerVerlag, Ch. Visco-plasticity, plasticity, creep and viscoplastic flow (problems of small, large and continuing deformation). 